\title{
Relação entre consumo de bebidas alcoólicas por universitárias e adiposidade corporal
}

\author{
Relationship between alcohol consumption by \\ female college students and body adiposity
}

Amanda Bertolini de Jesus Silva', Aihancreson Vaz Kirchoff de Oliveira², Janiara David Silva', Késia Diego Quintaes ${ }^{3}$, Vilma Aparecida da Silva Fonseca4 ${ }^{4}$, Aline Silva de Aguiar Nemer ${ }^{5}$

\section{RESUMO}

Objetivo: Identificar a influência do consumo de bebidas alcoólicas na adiposidade corporal de universitárias. Métodos: Realizou-se um estudo transversal com universitárias do curso de Nutrição da Universidade Federal de Ouro Preto (MG). O perfil de uso do álcool foi avaliado pelo AUDIT e a adiposidade foi avaliada pelo índice de massa corporal, percentual de gordura corporal, circunferência da cintura, circunferência do braço, dobra cutânea triciptal e razão cintura-quadril. Resultados: O consumo de bebidas foi relatado por 141 alunas, sendo que $56 \%(n=79)$ fazem uso nocivo de álcool (AUDIT $\geq 8$ ). Os valores médios do percentual de gordura corporal, da circunferência da cintura e da dobra cutânea triciptal foram maiores entre as alunas que relataram consumir bebidas alcoólicas $(p<0,05)$. Valores médios de circunferência do braço e dobra cutânea triciptal foram maiores entre as alunas que relataram uso nocivo de álcool. Houve associação positiva entre a pontuação do AUDIT e os parâmetros de adiposidade corporal (para percentual de gordura corporal, $r=0,20 ; p=0,03$ ); abdominal (para circunferência da cintura, $r=0,29 ; p=0,01$ ) e periférica (para circunferência do braço, $r=0,28 ; p=0,02$, e para dobra cutânea triciptal, $r=0,23$; $p=0,007)$. Conclusão: $O$ consumo de álcool pode ter influenciado na maior adiposidade entre as universitárias que relataram consumir bebidas alcoólicas. A adiposidade periférica foi maior entre as universitárias que relataram uso nocivo de álcool.

\section{ABSTRACT}

Objective: To identify the influence of alcohol intake on body adiposity of female college students. Methods: Female students attending the Nutrition College Course of Universidade Federal de Ouro Preto ( $M G$, Brazil) were the subjects of a transversal study. The pattern of alcohol consumption was evaluated by using the AUDIT questionnaire and the adiposity was evaluated by body mass index, percentage body fat, waist circumference, arm circumference, triceps skin-fold thickness and waist-to-hip ratio. Results: Alcohol consumption was reported by 141 students.

1 Universidade Federal de Ouro Preto (UFOP), Escola de Nutrição. 2 Universidade Federal de Ouro Preto (UFOP), Faculdade de Medicina. 


\section{Keywords}

Alcoholism, body

composition, nutritional

assessment.
Among them, harmful alcohol use (AUDIT $\geq 8$ ) was reported by $56 \%(n=79)$. Mean values of percentage body fat, waist circumference and triceps skin-fold thickness were higher among students who reported any alcohol consumption $(p<0.05)$. Values of arm circumference and triceps skin-fold thickness were increased among those with a drinking problem. There was a positive association between AUDIT scores and parameters of body adiposity (percentage body fat, $r=0.20 ; p=0.03$ ); abdominal adiposity (waist circumference, $r=0.29 ; p=0.01$ ) and peripheral adiposity (arm circumference, $r=0.28 ; p=0.02$, and triceps skin-fold thickness, $r=0.23 ; p=0.07$ ). Conclusion: Alcohol consumption may have increased the body adiposity among students reporting alcohol intake. Peripheral adiposity was higher among those in which this intake was harmful.

\section{INTRODUÇÃO}

Ao ingressar na universidade, o jovem é exposto a vários desafios, incluindo a separação da família e de amigos, maiores exigências acadêmicas, desenvolvimento de novas redes sociais e experimentação da ausência da supervisão de adultos $^{1}$. Aliado a isso, o maior consumo de álcool está relacionado ao acesso facilitado a essas bebidas nas frequentes festas universitárias e a busca da aceitação social².

O risco de desenvolver dependência e de sofrer consequências negativas relacionadas ao álcool eleva-se à medida que a frequência da intoxicação episódica aumenta ${ }^{3}$, especialmente quando há repetidos episódios de intoxicação, denominados binge ${ }^{4}$. O binge é caracterizado pelo consumo de quatro ou mais doses de álcool para as mulheres e cinco ou mais doses de álcool para os homens em uma única ocasião ${ }^{5}$. Entre universitários, observa-se a ampla ocorrência desses episódios, tornando essa população vulnerável aos efeitos deletérios do álcool ${ }^{6}$.

O etanol é a única droga psicoativa que fornece energia $(7,1 \mathrm{kcal} / \mathrm{g})$. Por ser fonte de calorias desprovidas de vitaminas e minerais, o consumo crônico de etanol causa desnutrição específica, além de promover a diminuição do consumo de alimentos e prejudicar a absorção dos nutrientes?. Entretanto, estudos com animais indicam que o consumo de doses baixas a moderadas de álcool promovem o ganho de peso de ratos desnutridos, sugerindo o aproveitamento de sua energia ${ }^{8,9}$.

Em humanos, Romeo et al. ${ }^{10}$ relataram que o consumo crônico e moderado de cerveja por um mês não modificou o peso nem a composição corporal de indivíduos adultos. Entretanto, estudos populacionais demonstraram que o aumento da gordura corporal e abdominal está relacionado ao consumo moderado ${ }^{11,12}$ e frequente ${ }^{13}$ de bebidas alcoólicas. Porém, ainda não há estudos na literatura que associem a gordura corporal e sua distribuição com o consumo de bebidas alcoólicas na população jovem universitária.

Assim, este trabalho teve como objetivo identificar a influência do consumo de bebidas alcoólicas na adiposidade corporal de universitárias.

\section{MÉTODOS}

Trata-se de um estudo epidemiológico transversal com universitárias do curso de Nutrição da Universidade Federal de Ouro Preto (UFOP), em Minas Gerais. O protocolo do estudo foi aprovado pelo Comitê de Ética em Pesquisa da UFOP (CAAE: 0002.0.238.000-09).

Todas as alunas acima de 18 anos e regularmente matriculadas foram convidadas a participar do estudo. Foram excluídos: os homens, devido ao restrito número de alunos matriculados no Curso de Nutrição da UFOP ( $n=11)$; as gestantes e as nutrizes, devido às modificações nos hábitos alimentares e no consumo de drogas. As universitárias que concordaram em participar do estudo assinaram o Termo de Consentimento Livre Esclarecido.

As estudantes preencheram um formulário com informações pessoais e responderam ao questionário AUDIT (do inglês The Alcohol Use Disorders Identification Test), validado para a população brasileira ${ }^{14}$. Ele é composto de dez perguntas que avaliam o consumo de álcool no último ano. Pontuação maior ou igual a 8 indica uso nocivo de bebidas alcoólicas.

Os questionários foram codificados pela equipe de pesquisa e sua aplicação foi feita em sala de aula, na ausência do docente, contando com a presença de dois aplicadores treinados.

A avaliação antropométrica foi realizada em data posteriormente agendada. Foram realizadas medidas antropométricas e de composição corporal para avaliar a distribuição de gordura. O peso e o percentual de gordura corporal (\%GC) foram determinados utilizando balança digital de bioimpedância elétrica bipolar (TANITA ${ }^{\oplus}$ BC554 Ironman) com os voluntários utilizando roupas leves e sem sapatos. A estatura foi medida nas mesmas condições utilizando estadiômetro (Altura Exata) ${ }^{15}$.

O estado nutricional foi avaliado pelo índice de massa corporal (IMC) calculado pela equação de Quetelet (IMC = peso/altura²) e interpretado conforme a Organização Mundial da Saúde $(\mathrm{OMS})^{16}$.

A dobra cutânea tricipital (DCT) foi realizada no hemicorpo direito do indivíduo e sendo a dobra aferida pelo adi- 
pômetro (LANGE ${ }^{\oplus}$ ) a $1 \mathrm{~cm}$ abaixo do ponto pinçado pelos dedos. O resultado foi expresso em média de três medidas da mesma dobra ${ }^{17}$.

As circunferências corporais foram realizadas com o avaliador em pé e estando a fita métrica flexível em ângulo reto em relação ao eixo do corpo ${ }^{15}$. A circunferência do braço (CB) foi aferida no ponto médio entre o acrômio e o olecrano. O resultado foi interpretado conforme valores de referência ${ }^{18}$. A circunferência da cintura (CC) foi realizada com o indivíduo em pé com a fita circundando o ponto médio entre a última costela e a crista ilíaca e a leitura feita no momento da expiração ${ }^{16}$. A circunferência do quadril (CQ) foi medida com a fita métrica flexível circundando a região de maior perímetro entre a cintura e a coxa, no nível da região glútea. Calculou-se a razão cintura-quadril (RCQ) pela equação $R C Q=C C / C Q^{19}$.

A adiposidade foi avaliada pelo \%GC (adiposidade corporal), DCT e CB (adiposidade periférica) e CC e RCQ (adiposidade abdominal).

\section{Análise estatística}

Os dados foram analisados pelo SPSS 12.0. Os resultados foram expressos em média \pm desvio-padrão. Após avaliação da normalidade pelo teste de Kolmogorov-Smirnov, utilizou-se o teste $t$ de Student (CB, CC e RCQ) ou teste de U-Mann Whitney (IMC, DCT e \%GC) para comparação entre universitárias que consomem bebidas alcoólicas com as que não as consomem. Utilizou-se ANOVA one way seguido do teste de Bonferroni para comparação entre as universitárias que não consomem bebidas alcoólicas, AUDIT $<8$ e AUDIT $\geq 8$ quanto a CB, CC e RCQ. O teste de Kruskall Wallis seguido do U-Mann Whitney foi utilizado para comparação entre os grupos quanto ao IMC, \%GC e DCT. Associações entre a pontuação do AUDIT e as medidas antropométricas foram avaliadas pelo coeficiente de correlação de Pearson e Spermann. O nível de significância foi fixado em 5\%.

\section{RESULTADOS}

Participaram da pesquisa 178 alunas com idade média de $21,5 \pm 1,96$ anos. A maior parte das estudantes seguia alguma religião $(90,9 \%)$ e recebia ajuda financeira da família (88,3\%) e/ou da universidade (55,1\%). Quanto à moradia, $54,8 \%$ moravam em repúblicas particulares, 24,3\%, em casa de familiares, 10,2\%, em repúblicas federais e os demais moravam sozinhos, em pensão ou alojamento.

Das 141 universitárias que relataram consumir bebidas alcoólicas, $56 \%$ ( $n=79$ ) o faziam de forma nociva (AUDIT $\geq 8$ ). Setenta e sete alunas $(54,6 \%)$ consumiram cinco doses ou mais de bebidas por ocasião (binge), com frequência semanal $(n=38)$ ou menos do que semanal $(n=74)$.

Das 178 alunas que preencheram os questionários, 117 realizaram a avaliação antropométrica, sendo que 45 justifi- caram sua ausência. O desinteresse pela pesquisa foi o motivo de desistência de 21 alunas (46,7\%), 14 alunas $(31,1 \%)$ tinham horários incompatíveis com os da avaliação, a vergonha do peso foi o motivo de desistência de 8 alunas $(17,8 \%)$ e 2 alunas (4,4\%) abandonaram o curso.

As universitárias que relataram consumir bebidas alcoólicas apresentaram maiores resultados de medidas de adiposidade corporal (\%GC), abdominal (CC) e periférica (DCT) em comparação às que relataram não consumir bebidas alcoólicas (Figura 1). O IMC não diferiu entre as universitárias que relataram consumir ou não bebidas alcoólicas, assim como as medidas de CB e RCQ (Tabela 1). A maioria das alunas que relatou consumir ou não bebidas alcoólicas encontrava-se eutrófica, sendo $83,3 \%$ e $81,6 \%$, respectivamente.

Houve associação positiva entre a pontuação do AUDIT e as medidas de IMC $(r=0,23 ; p=0,01)$; adiposidade corporal (\%GC, $r=0,20, p=0,03$ ), periférica (DCT, $r=0,25 ; p=0,007$ ) $C B, r=0,28 ; p=0,002)$ e abdominal $(C C, r=0,29, p=0,01)$ (Figura 2).

Comparando as universitárias que não consomem bebidas alcoólicas com as que fazem uso baixo a moderado de álcool (AUDIT < 8) e as que o fazem de forma prejudicial (AUDIT $\geq 8$ ), houve diferença estatística significativa apenas para os parâmetros de adiposidade periférica (CB e DCT), indicando maior média entre aquelas com AUDIT $\geq 8$ (Tabela 2).

Tabela 1. Medidas antropométricas das universitárias que relataram consumir ou não bebidas alcoólicas

\begin{tabular}{lcc}
\hline & $\begin{array}{c}\text { Consomem bebidas alcoólicas } \\
(\mathbf{n}=93)\end{array}$ & $\begin{array}{c}\text { Não consomem bebidas } \\
\text { alcólicas } \\
(\mathbf{n}=24)\end{array}$ \\
\hline IMC $\left(\mathrm{kg} / \mathrm{m}^{2}\right)$ & $21,6 \pm 2,63$ & $20,6 \pm 2,34$ \\
CB (cm) & $27,3 \pm 7,56$ & $25,6 \pm 2,95$ \\
RCQ & $0,71 \pm 0,04$ & $0,72 \pm 0,06$ \\
\hline
\end{tabular}

$p>0,05$ (não significativo) para todos os parâmetros antropométricos quando comparadas as universitárias que consomem com as que não consomem bebidas alcoólicas.

IMC: índice de massa corporal; CB: circunferência do braço; RCQ: razão cintura-quadril.

Tabela 2. Medidas antropométricas e de composição corporal das universitárias que não consomem bebidas alcoólicas, com AUDIT $<8$ e AUDIT $\geq 8$ pontos

\begin{tabular}{lcccc}
\hline & $\begin{array}{c}\text { Não consomem } \\
\text { bebidas alcoólicas } \\
(\mathrm{n}=24)\end{array}$ & $\begin{array}{c}\text { AUDIT }<8 \\
(\mathrm{n}=47)\end{array}$ & $\begin{array}{c}\text { AUDIT } \geq 8 \\
(\mathrm{n}=46)\end{array}$ & $\mathbf{p}^{\mathbf{a}}$ \\
\hline $\mathrm{GC}(\%)$ & $21,52 \pm 8,37$ & $23,54 \pm 5,92$ & $24,83 \pm 6,28$ & 0,143 \\
$\mathrm{IMC}\left(\mathrm{kg} / \mathrm{m}^{2}\right)$ & $20,92 \pm 2,36$ & $21,10 \pm 2,57$ & $21,88 \pm 2,69$ & 0,225 \\
$\mathrm{CB}(\mathrm{cm})$ & $25,79 \pm 2,97$ & $25,70 \pm 2,66^{*}$ & $27,20 \pm 3,24^{*}$ & 0,030 \\
$\mathrm{CC}(\mathrm{cm})$ & $66,14 \pm 12,04$ & $68,81 \pm 5,77$ & $70,65 \pm 6,13$ & 0,065 \\
RCQ & $0,71 \pm 0,06$ & $0,70 \pm 0,04$ & $0,71 \pm 0,05$ & 0,777 \\
DCT $(\mathrm{mm})$ & $20,16 \pm 6,25^{* *}$ & $21,02 \pm 5,02^{*}$ & $23,69 \pm 5,54^{* *}$ & 0,037 \\
\hline
\end{tabular}

${ }^{a}$ Teste de Kruskall-Wallis; ${ }^{*} p<0,05$, diferença entre os grupos AUDIT $<8$ X AUDIT $\geq 8$ (Teste U-Mann Whitney); * $\mathrm{p}<0,05$, diferença entre os grupos não consomem bebidas alcoólicas $x$ AUDIT $\geq 8$.

GC: gordura corporal; IMC: índice de massa corporal; CB: circunferência do braço; CC: circunferência da cintura; RCQ: razão cintura-quadril; DCT: dobra cutânea triciptal. 

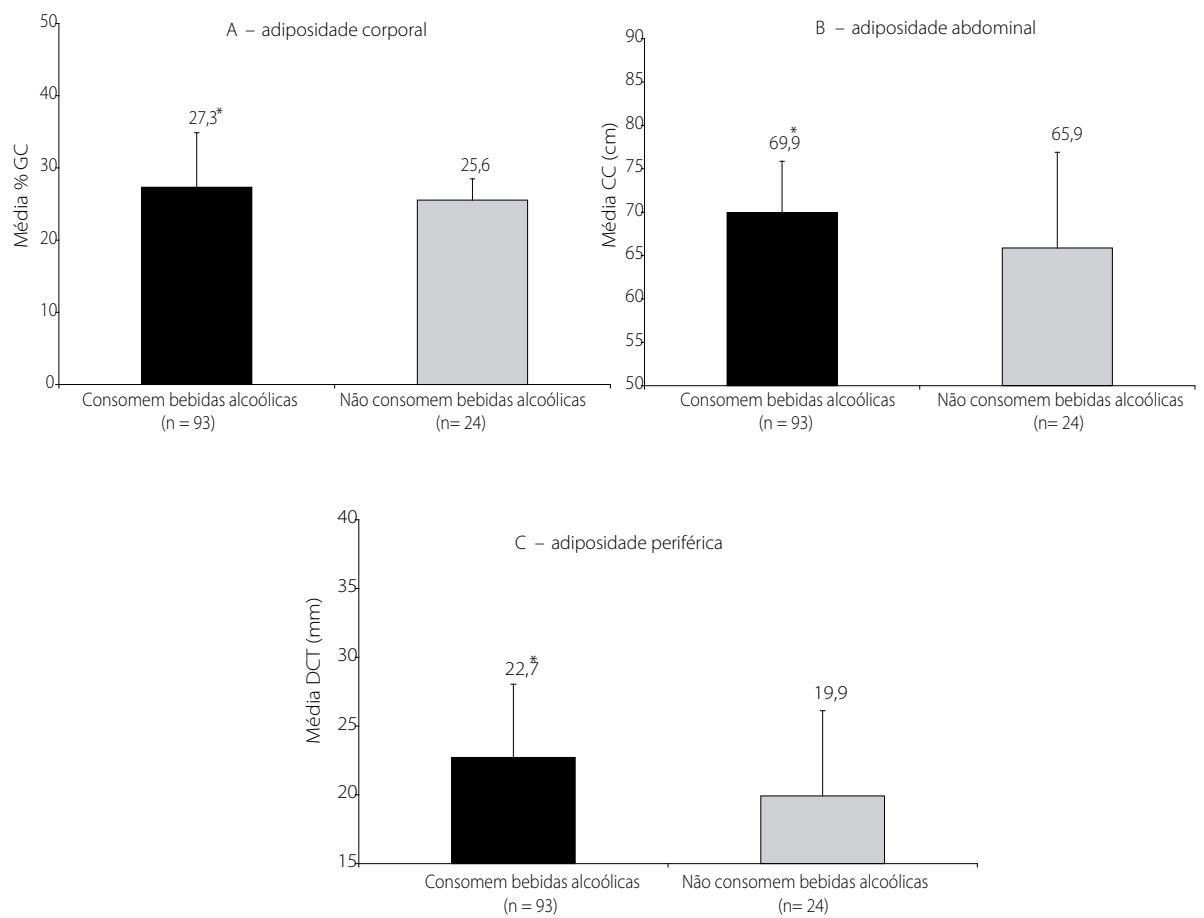

${ }^{*} \mathrm{p}<0,05$ quando comparadas às universitárias que consomem bebidas alcoólicas com as que não consomem bebidas alcoólicas. $\% G C$ : percentual de gordura corporal; $C$ : circunferência da cintura; DCT: dobra cutânea triciptal.

Figura 1. Adiposidade corporal, abdominal e periférica das universitárias que relataram consumir ou não bebidas alcoólicas.
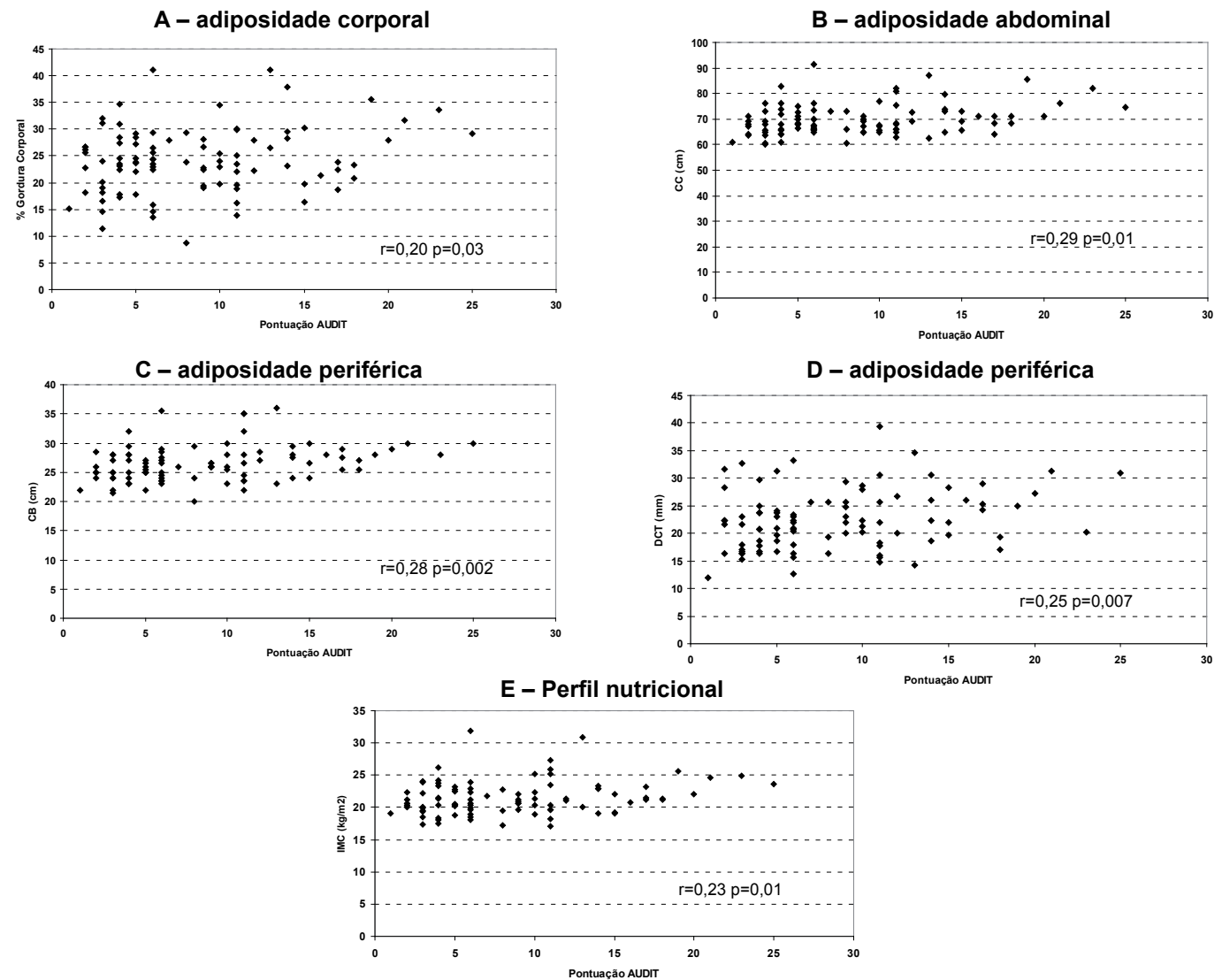

r: coeficiente de correlação (correlaçăo de Pearson: CB, CC e RCQ; correlação de Spearman: IMC, DCT e \%GC).

IMC: índice de massa corporal; \%GC: percentual de gordura corporal; CC: circunferência da cintura; CB: circunferência do braço; DCT: dobra cutânea triciptal.

Figura 2. Correlação entre a pontuação do AUDIT e os parâmetros antropométricos de adiposidade corporal (A), adiposidade abdominal (B) e adiposidade periférica (C e D) e perfil nutricional (E), 


\section{DISCUSSÃO}

O estudo demonstrou que a maioria das universitárias estava classificada como eutrófica pelo perfil nutricional avaliado pelo IMC. Entretanto, as universitárias que relataram consumir bebidas alcoólicas apresentaram maiores médias dos parâmetros antropométricos de avaliação de adiposidade corporal (\%GC), abdominal (CC) e periférica (CB e DCT).

O etanol fornece calorias em seu metabolismo $(7,1$ $\mathrm{kcal} / \mathrm{g}$ ), sendo uma fonte energética intermediária em relação às proteínas e aos carboidratos, que fornecem 4,0 kcal/g, e aos lipídios, que fornecem $9,0 \mathrm{kcal} / \mathrm{g}$. O aproveitamento orgânico da energia fornecida pelas bebidas alcoólicas dependerá do estado nutricional, da frequência e do modo de consumo, podendo levar ao sobrepeso e à obesidade, em consumidores moderados, e à desnutrição entre os usuários crônicos $8,20,21$.

A enzima ADH, presente no citoplasma de hepatócitos, é a principal via hepática para a oxidação do álcool consumido em baixas a moderadas quantidades. A ingestão de doses altas de álcool aciona a segunda via hepática de metabolização, o sistema microssomal de oxidação do etanol (MEOS). Para os consumidores moderados de álcool, as calorias provenientes do etanol são obtidas pelo metabolismo hepático da droga por meio da enzima ADH. Por essa via, há produção de acetaldeído por meio da transferência de hidrogênio para o cofator nicotinamida adenina dinucleotídeo (NAD), reduzindo-o a NADH. Essa reação está associada a um alto fornecimento energético proveniente da oxidação do álcool e formação de 16 ATP/mol via fosforilação oxidativa22,23. Saturando a via ADH, a via MEOS é ativada para oxidar o excesso de etanol consumido, porém com gasto energético e sem formação de componentes geradores de energia, como o $\mathrm{NADH}^{23}$.

Por ser uma substância tóxica, o álcool tem prioridade no metabolismo hepático alterando outras vias metabólicas, incluindo a diminuição da oxidação lipídica, levando a um balanço positivo de gorduras ${ }^{23,24}$. O consumo moderado a alto de álcool (aproximadamente $30 \mathrm{~g}$ de etanol/dia) está relacionado ao descontrole metabólico e hormonal, com implicações na homeostase energética, aumento do apetite e ganho de peso corporal ${ }^{25}$.

Entre os bebedores moderados, as calorias provenientes do consumo de bebidas alcoólicas são adicionadas à dieta habitual. Entre os dependentes, as calorias provenientes do álcool podem representar 50\% das suas necessidades, substituindo o consumo de alimentos como fonte de energia e de nutrientes, ocorrendo emagrecimento e desnutrição ${ }^{20}$.

No presente estudo, mais da metade das universitárias relatou uso nocivo de bebidas alcoólicas (AUDIT $\geq 8$ ). Também foi alta a prevalência do consumo de bebidas em binge (56,8\%). É preocupante a alta prevalência de uso nocivo de bebidas alcoólicas identificado em um curso de graduação composto em sua maioria por mulheres. É conhecido que as mulheres apresentam ação reduzida da enzima álcool desidrogenase gástrica e maior \%GC. Esses fatores favorecem a maior alcoolemia, intoxicação e desenvolvimento de doenças hepáticas alcoólicas, mesmo quando o consumo de álcool é similar ao do sexo oposto ${ }^{26}$. Associado aos danos orgânicos provenientes do consumo de álcool entre as muIheres, a ingestão de álcool favorece o maior consumo energético, levando ao risco de obesidade e de desenvolvimento de doenças crônicas relacionadas ao aumento da gordura corporal e abdominal.

Entre as universitárias avaliadas, houve aumento dos parâmetros antropométricos de adiposidade corporal (\%GC), abdominal (CC) e periférica (DCT). Entretanto, a comparação dos resultados do presente estudo com outros da literatura fica comprometida devido à ausência de estudos avaliando o consumo de álcool, ganho de peso e alteração na adiposidade entre a população jovem universitária. No Brasil, apenas Ferreira et al..$^{27}$ avaliaram a adiposidade corporal (\%GC) e abdominal (CC e RCQ) de doadores de sangue com idades entre 20 a 59 anos que consumiam bebidas alcoólicas. Os autores não relataram a variação da adiposidade por faixa etária, mas identificaram que a cintura e a RCQ mantiveram-se associadas positivamente com o consumo total de álcool e semanal de cerveja.

Estudos epidemiológicos internacionais também encontraram associação entre o aumento do consumo de álcool e o aumento da adiposidade corporal e abdominal|28,29, independente do tipo de bebida e se esta é consumida ou não com as refeições ${ }^{30}$. Tolstrup et al. ${ }^{12}$ relataram que a frequência de consumo de bebidas alcoólicas foi mais fortemente associada com o aumento da CC do que a quantidade ingerida de álcool. Além da adição calórica do álcool, seu consumo favorece o aumento da secreção de cortisol. O aumento da secreção desse hormônio está relacionado ao acúmulo de gordura abdominal ${ }^{22}$.

No presente estudo, a maioria das alunas encontrava-se eutrófica segundo o perfil nutricional avaliado pelo IMC, apesar da maior adiposidade entre as consumidoras de bebidas alcoólicas. Possivelmente, as alunas que não se consideravam dentro dos padrões estabelecidos pela mídia recusaram-se a continuar na pesquisa realizando a avaliação antropométrica. Esse foi um fator de limitação do estudo que comprometeu o número total de participantes para a associação da adiposidade relacionada ao aumento da pontuação do AUDIT. Outra limitação do estudo foi não ter avaliado o consumo energético proveniente da alimentação, das bebidas alcoólicas e o gasto energético decorrente da prática de atividade física, fatores que podem interferir na adiposidade corporal.

Além de concordar com dados da literatura sobre o aumento da adiposidade entre consumidoras de bebidas alcoólicas, o estudo foi capaz de identificar o aumento da adiposidade periférica ou subcutânea, principalmente entre as 
que fizeram uso nocivo de álcool. A avaliação de gordura periférica relacionada ao consumo de álcool não foi relatada na literatura, apenas a avaliação do IMC, gordura corporal e CC.

Deve-se considerar que essas universitárias ainda são jovens e que, apesar da diferença quanto ao acúmulo de gordura corporal, esses valores ainda não mostram risco para o desenvolvimento de doenças cardiovasculares. Porém, o contexto em que essas alunas estão inseridas, a continuidade do consumo de bebidas e o acréscimo energético proveniente desse consumo podem favorecer o desenvolvimento de dependência química e de doenças crônicas relacionadas ao acúmulo de gordura corporal e visceral.

\section{CONCLUSÃO}

Os resultados indicam que as jovens universitárias consumidoras de bebidas alcoólicas apresentaram maior acúmulo de gordura corporal (\%GC), abdominal (CC) e periférica (DCT e CB). Houve associação positiva entre o aumento da pontuação do AUDIT e o aumento dos valores de IMC, \%GC, CC, DCT e CB.

\section{AGRADECIMENTOS}

À Roberta R. R. Álvares, pelo auxílio na coleta de dados, à Pró-Reitoria de Pesquisa e Pós-graduação da UFOP, pelo apoio financeiro. Amanda Bertolini de Jesus Silva agradece o auxílio do Ministério da Educação (MEC), por meio do PET-Nutrição, e Késia Diego Quintaes agradece à Fundação de Amparo à Pesquisa do Estado de Minas Gerais (FAPMIG) pelo apoio financeiro.

\section{REFERÊNCIAS}

1. Peuker AC, Fogaça J, Bizarro L. Expectativas e beber problemático entre universitários. Rev Psic Teor Pesq. 2006;22(2):193-200.

2. Dimeff $L A$, Baer JS, Kivlahan DR, Marlatt GA. Alcoolismo entre estudantes universitários: uma abordagem de redução de danos. São Paulo: Unesp; 2002.

3. Wechsler H, Molnar BE, Davenport AE, Baer J. College alcohol use: a full or empty glass? J Am Coll Health. 1999;47(6):247-52.

4. Shakeshaft AP, Bowman JA, Sanson-Fisher RW. Comparison of three methods to assess binge consumption: one-week retrospective drinking diary, AUDIT, and quantity/frequency. Subst Abus. 1998;19(4):191-203.

5. Naimi TS, Brewer RD, Mokdad A, Denny C, Serdula MK, Marks IS. Binge drinking among US adults. JAMA. 2003;289(1):70-5.

6. Sheffield FD, Darkes J, Del Boca FK, Goldman MS. Binge drinking and alcohol-related problems among community college students: implications for prevention policy. Am J Coll Health. 2005;54(3):137-41.
7. Molina PE, Hoek JB, Nelson S, Guidot DM, Lang CH, Wands JR. Mechanisms of alcohol-induced tissue injury. Alcohol Clin Exp Res. 2003;27(3):563-75.

8. Aguiar AS, Da-Silva VA, Boaventura GT. Can calories from ethanol contribute to body weight preservation by malnourished rats? Braz J Med Biol Res. 2004;37(6):841-46.

9. Aguiar AS, Boaventura GT, Abrahão RF, Freitas TL, Takiya CM, Soares-Filho PJ, et al. Ethanol in low chronic dose level attenuates major organic effects in malnourished rats. Biol Res. 2009:42(1):31-40.

10. Romeo J, Gonzáles-Gross M, Wärnberg J, Díaz LE, Marcos A. ¿Influye la cerveza en el aumento de peso? Efectos de un consumo moderado de cerveza sobre la composición corporal. Nutr Hosp. 2007;22(2):223-8.

11. Dorn JM, Hovey K, Muti P. Alcohol drinking patterns differentially affect abdominal adiposity as measured by abdominal height in women and men. J Nutr. 2003;133(8):2655-62.

12. Tolstrup JS, Heitmann BL, Tjonneland A, Overvad K, Sorensen TI, Gronbaek M. The relation between drinking pattern and body mass index, waist and hip circumference. Int J Obes. 2005;29:490-7.

13. Tolstrup JS, Halkjaer J, Heitmann BL, Tjønneland AM, Overvad K, Sørensen TIA, et al. Alcohol drinking frequency in relation to subsequent changes in waist circumference. Am J Clin Nutr. 2008;87(4):957-63.

14. Lima CT, Freire ACC, Silva APB, Teixeira RM, Farrel M, Prince M. Concurrent and construct validity of the audit in an urban brazilian sample. Alcohol. 2005;40(6):584-9.

15. Guedes DP. Composição corporal: princípios, técnicas e aplicações. Santa Catarina: CEITEC; 1998

16. Organização Mundial da Saúde (OMS). Obesity - Presenting and managing the global epidemic. Report of a WHO consultation on obesity. Genebra: OMS; 1998.

17. Durnin JVGA, Womersley J. Body fat assessed from total body density and its estimation from skinfold thickness: measurements on 481 men and women aged from 16 to 71 years. Brit J Nut. 1974;32(1):77-97.

18. Frisancho AR. New norms of upper limb fat and muscle areas for assessment of nutritional status. Am J Clin Nutr. 1981;34(11):2540-5.

19. Pouliot MC, Despres JP, Lemieux S, Moorjani S, Bouchard C, Tremblay A, et al. Waist circumference and abdominal saggital diameter: best simple anthropometric indexes of abdominal visceral adipose tissue accumulation and related cardiovascular risk in men and women. Am J Cardiol. 1994;73(7):460-8.

20. Colditz GA, Giovannucci E, Rimm EB, Stampfer MJ, Rosner B, Speizer FE, et al. Alcohol intake in relation to diet and obesity in women and men. Am J Clin Nutr. 1991;54(1):49-55.

21. Aguiar AS, Silva VA, Boaventura GT. As calorias do etanol são aproveitadas pelo organismo? Nutr Pauta. 2007;15:45-9.

22. Suter PM, Häsler E, Vetter W. Effects of alcohol on energy metabolism and body weight regulation: is alcohol a risk factor for obesity? Nutr Rev. 1997;55(5):157-71.

23. Lieber CS. Perspectives: do alcohol calories count? Am J Clin Nutr. 1991;54(6):976-82.

24. Suter PM. Is alcohol consumption a risk factor for weight gain and obesity? Crit Rev Clin Lab Sci. 2005;42(3):197-227.

25. Pimentel GD, Bressan JB. 0 consumo de álcool altera os hormônios reguladores do apetite, aumentando a fome e o peso corporal. Rev Bras Nutr Clin. 2010;25(1):83-90.

26. Fuchs CS, Stampfer MJ, Colditz GA, Giovannucci El, Manson JE, Kawachi I, et al. Alcohol consumption and mortality among women. N Engl J Med. 1995;332(19):1245-50.

27. Ferreira MG, Valente JG, Gonçalves-Silva RMV, Sichieri R. Consumo de bebida alcoólica e adiposidade abdominal em doadores de sangue. Rev Saúde Pública. 2008;42(6):1067-73.

28. Rissanen AM, Heliovaara M, Knekt P, Reunanen A, Aromaa A. Determinants of weight gain and overweight in adult Finns. Eur J Clin Nutr. 1991;45(9):419-30.

29. Wannamethee SG, Shaper AG. Alcohol, body weight, and weight gain in middle-aged men. Am J Clin Nutr. 2003;77(5):1312-7.

30. Wannamethee SG, Shaper AG, Whincup PH. Alcohol and adiposity: effects of quantity and type of drink and time relation with meals. Int J 0bes. 2005;29(2):1436-44. 